\title{
Transcendental operators acting on slice regular functions
}

https://doi.org/10.1515/conop-2022-0002

Received October 31, 2021; accepted January 3, 2022

\begin{abstract}
The aim of this paper is to carry out an analysis of five trascendental operators acting on the space of slice regular functions, namely *-exponential, *-sine and *-cosine and their hyperbolic analogues. The first three of them were introduced by Colombo, Sabadini and Struppa and some features of ${ }^{\star}$-exponential were investigated in a previous paper by Altavilla and the author. We show how $\exp _{\star}(f), \sin _{\star}(f), \cos _{\star}(f), \sinh _{\star}(f)$ and $\cosh _{*}(f)$ can be written in terms of the real and the vector part of the function $f$ and we examine the relation between $\cos \star$ and $\cosh \star$ when the domain $\Omega$ is product and when it is slice. In particular we prove that when $\Omega$ is slice, then $\cos \star(f)=\cosh *\left({ }^{\star} I\right)$ holds if and only if $f$ is $\mathbb{C}_{I}$ preserving, while in the case $\Omega$ is product there is a much larger family of slice regular functions for which the above relation holds.
\end{abstract}

Keywords: Slice-regular functions, *-product of slice-regular functions, *-exponential, * sine and cosine, *logarithm

MSC: Primary 47J05; secondary: 30G35, 39B52

\section{Introduction}

In this paper we investigate the behaviour of some operators defined on the space of slice regular functions. They arise as natural analogues of the corresponding ones in the complex holomorphic setting, but the issues about the composition of slice regular functions entail some unexpected behaviour and at the same time they open a large class of interesting questions to be answered.

After recalling the necessary definitions, in Section 2 we show some preliminary results which generalize to the quaternionic setting the usual relations between exponential, sine and cosine and their hyperbolic analogues which hold in the complex case. Moreover, in Proposition 2.19 we are able to express the value of these operators on a function $f$ in terms of its real and vector parts, by means of the functions $\mu$ and $v$ which were introduced in [2]. In particular, these relations allow us to show that ${ }^{*}$-sine and ${ }^{*}$-cosine still satisfy an analogue of the Pytagorean Trigonometric Identity, see Proposition 2.20. In Section 3 we carry out an analysis on the slice-regular functions $\mu$ and $\nu$ which are needed to deal with *-exponential, *-sine and *-cosine and their hyperbolic analogues. We are thus able to characterize when, given $c \in \mathbb{R}, \mu(q)= \pm \mu(q+c)$ or $\mu(q)= \pm \mu(c-q)$ (see Proposition 3.3) and to classify all functions which solve a sine-cosine problem, thus concluding in Proposition 3.4 the analysis carried out in [2, Proposition 4.6].

Last section contains a detailed analysis of the relations which hold between *-cosine and hyperbolic ${ }^{*}$-cosine. As a first result we prove that given a product domain $\Omega$ then $\cos *(f)=\cosh *(\mathcal{J} f)$ is true for any slice regular function $f$, where $\mathcal{J}$ is the function introduced in Definition 2.3. When the domain $\Omega$ is slice, the function $\mathcal{J}$ cannot be defined anymore, so that an analogue of the relation in the complex case is given by $\cos *(f)=\cosh _{*}\left(f^{\star} I\right)$, where $I \in \mathbb{S}$ is an imaginary unit. We first prove in Proposition 4.2 that this relation holds

\footnotetext{
*Corresponding Author: Chiara de Fabritiis: Dipartimento di Ingegneria Industriale e Scienze Matematiche, Università Politecnica delle Marche, Via Brecce Bianche, 60131, Ancona, Italia, E-mail: fabritiis@dipmat.univpm.it GNSAGA of INdAM
} 
for any $f$ slice regular and $\mathbb{C}_{I}$-preserving, then an accurate study of the behaviour of ${ }^{\star}$-cosine and hyperbolic

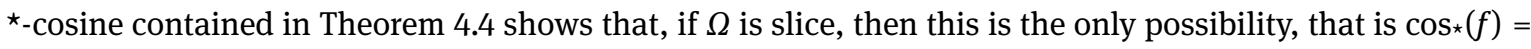
$\cosh _{\star}(f \star I)$ implies that $f$ is $\mathbb{C}_{I}$-preserving.

At last, we deal with the case when the domain $\Omega$ is product, here the statement of Theorem 4.5 gives a complete list of all slice regular functions $f$ which satisfy the equality $\cos \star(f)=\cosh _{\star}(f \star I)$. In particular, in addition to $\mathbb{C}_{I}$ preserving functions there are several classes of slice regular functions defined on $\Omega$ which satisfy the above equality, e.g., all the functions of the form $f=2\left(f_{0}+f_{2} J\right) * \ell_{I}^{ \pm}$, where $f_{0}$ and $f_{2}$ are slicepreserving, $J \in \mathbb{S}$ is orthogonal to $I$ and $\ell_{I}^{ \pm}$are the idempotent functions introduced in Definition 2.3.

\section{Definitions and preliminary results}

Slice regular functions on quaternions $\mathbb{H}$ were introduced by Gentili and Struppa some 15 years ago, see [11] and [12], in order to give a suitable generalization of the notion of holomorphic function to domains contained in $\mathbb{H}$. We recall that the set of imaginary quaternions $\operatorname{Im} \mathbb{H} \simeq \mathbb{R}^{3}$ contains the elements of $\mathbb{H}$ whose real part is zero, while the set $\mathbb{S}=\left\{q \in \mathbb{H}: q^{2}=-1\right\}$ is a subset of $\operatorname{Im} \mathbb{H}$ which is often called the sphere of imaginary units since it is isomorphc to the sphere $S^{2} \subset \mathbb{R}^{3}$. In the sequel, we will always deal with slice regular functions defined on circular domains, where a domain $\Omega \subseteq \mathbb{H}$ is said to be circular if $x+y I \in \Omega$ for a certain $I \in \mathbb{S}$ implies $x+y J \in \Omega$ for any $J \in \mathbb{S}$. A circular domain is said to be slice if $\Omega \cap \mathbb{R} \neq \emptyset$, otherwise it said to be product.

When the domain $\Omega$ is slice, we can define slice regular functions as functions which satisfy the CauchyRiemann equation on any slice. For any $I \in \mathbb{S}$ and $f$ defined on $\Omega$, we denote by $\Omega_{I}$ the intersection $\Omega \cap \mathbb{C}_{I}$, where $\mathbb{C}_{I}=\operatorname{Span}_{\mathbb{R}}(1, I)$ and by $f_{I}$ the restriction of $f$ to $\Omega_{I}$.

Definition 2.1. Let $\Omega \subseteq \mathbb{H}$ be a slice domain. A function $f: \Omega \rightarrow \mathbb{H}$ is said to be slice regular if for any $I \in \mathbb{S}$, its restriction $f_{I}$ has continuous partial derivatives on $\Omega_{I}$ and

$$
\bar{\partial}_{I} f(x+y I)=\frac{1}{2}\left(\frac{\partial}{\partial x}+I \frac{\partial}{\partial y}\right) f_{I}(x+y I) \equiv 0 .
$$

If the domain $\Omega$ is product, functions which satisfy the Cauchy Riemann equation on any slice could not be continuous, so in this case the above definition is not the appropriate one any more. The extension of the notion of slice regular function to the case of product domains requires the introduction of the formalism of stem functions, whose theory was established by Ghiloni and Perotti in [15] in order to deal with slice regular functions defined on product domains.

In the following we will denote the space of slice regular functions on $\Omega$ by $\mathcal{S R}(\Omega)$. The following two classes of regular functions arise naturally in the development of the theory.

Definition 2.2. Given $f \in \mathcal{S R}(\Omega)$ and $I \in \mathbb{S}$, we say that $f$ is $\mathbb{C}_{I}$-preserving if $f\left(\Omega_{I}\right) \subset \mathbb{C}_{I}$; we say that $f$ is slice preserving if it is $\mathbb{C}_{I}$-preserving for all $I \in \mathbb{S}$.

For any $I \in \mathbb{S}$, the set of slice preserving functions and of $\mathbb{C}_{I}$-preserving functions defined on $\Omega$ will be denoted by $\mathcal{S} \mathcal{R}_{\mathbb{R}}(\Omega)$ and $\mathcal{S} \mathcal{R}_{I}(\Omega)$, respectively. For a detailed study of functions which preserve either one or all slices, see [3].

Definition 2.3. We denote by $\mathcal{J}$ the slice regular, slice preserving function $\mathcal{J}: \mathbb{H} \backslash \mathbb{R} \rightarrow \mathbb{S}$ given by $\mathcal{J}(x+y I)=I$ for any $y>0$. For any $I \in \mathbb{S}$ we set $\ell_{I}^{ \pm}=\frac{1}{2}(1 \mp J I)$; it is easily seen that $\ell_{I}^{ \pm}$are $\mathbb{C}_{I}$-preserving functions.

The functions $\ell_{I}^{ \pm}$are idempotents in $\mathcal{S R}(\Omega)$ with respect to the *-product which we are going to define on $\mathcal{S R}(\Omega)$; they were introduced in [15] and they are thoroughly studied in [7], [4] and [5].

Either by using the Splitting Lemma and the Extension Principle (see [13], pp. 10-12) or the stem machinery, we can define a product, called the ${ }^{\star}$-product (or slice product in the more general context of slice 
functions), on $\mathcal{S R}(\Omega)$ which gives this set the structure of an associative algebra. As a matter of fact, in general the pointwise product of two slice regular functions is no more regular, so a different product is needed in order to obtain a valid multiplicative structure. The following proposition describes the *-product in a different way, for a proof see [13] if $\Omega$ is slice and [1] if $\Omega$ is product.

Proposition 2.4. Let $f, g \in \mathcal{S R}(\Omega)$, then

$$
(f \star g)(q)= \begin{cases}0 & \text { if } f(q)=0, \\ f(q) g\left(f(q)^{-1} q f(q)\right) & \text { if } f(q) \neq 0 .\end{cases}
$$

Remark 2.5. If $f$ is $\mathbb{C}_{I}$-preserving, then we have $(f \star g)_{I}=f_{I} g_{I}$ for any slice regular function $g$. Indeed, if $q \in \Omega_{I}$ and $f(q)=0$, the relation is trivial. Otherwise, if $q \in \mathbb{C}_{I}$ we have $\left(f^{\star} g\right)(q)=f(q) g\left(f(q)^{-1} q f(q)\right)$; since $f(q)$ and $q$ both belong to $\mathbb{C}_{I}$ they do commute, and thus $\left(f^{\star} g\right)(q)=f(q) g(q)$. Moreover, if both $f$ and $g$ are $\mathbb{C}_{I}$-preserving, then $f^{\star} g=g \star f$, as $(f \star g)_{I}=f_{I} g_{I}=g_{I} f_{I}=(g \star f)_{I}$ and we can apply the Identity Principle (see [13, Theorem 1.12] and the analogous result in [1]).

The structure of $\mathcal{S R}(\Omega)$ is elucidated by the following result, originally due to Colombo, Gonzalez-Cervantes and Sabadini and then generalized by Ghiloni, Moretti and Perotti (see [8] and [14]).

Let $1, I, J, K$ be a basis of $\mathbb{H}$.

Proposition 2.6. The map

$$
\left(\mathcal{S R}_{\mathbb{R}}(\Omega)\right)^{4} \ni\left(f_{0}, f_{1}, f_{2}, f_{3}\right) \mapsto f_{0}+f_{1} I+f_{2} J+f_{3} K \in \mathcal{S R}(\Omega)
$$

is bijective. In particular, for any $f \in \mathcal{S R}(\Omega)$ there exists a unique four-tuple $f_{0}, f_{1}, f_{2}, f_{3} \in \mathcal{S R}_{\mathbb{R}}(\Omega)$ such that $f=f_{0}+f_{1} I+f_{2} J+f_{3} K$. Moreover, if $I \in \mathbb{S}$ then $f$ is $\mathbb{C}_{I}$-preserving iff $f_{2}=f_{3} \equiv 0$.

We underline that the functions $f_{0}, \ldots, f_{3}$ in the statement of the above proposition are slice-preserving which does not mean they are real-valued. From now on we will always consider $1, I, J, K$ to be an orthonormal basis of $\mathbb{H}$.

Definition 2.7. Let $f=f_{0}+f_{1} I+f_{2} J+f_{3} K$. We call $f_{0} \in \mathcal{S} \mathcal{R}_{\mathbb{R}}(\Omega)$ the real part of $f, f_{V}=f_{1} I+f_{2} J+f_{3} K$ the vector part of $f, f^{c}=f_{0}-f_{v}$ the conjugate of $f$ and $f^{s}=f^{c} \star f=f^{\star} f^{c}$ the symmetrized function of $f$.

Since the basis $1, I, J, K$ is orthonormal, all these definitions are intrinsic, that is, they do not depend on the choice of the basis but on the function $f$ only (indeed, this is true when I,J,K, belong to $\operatorname{Im} \mathbb{H}$ ).

According to the fact that $\Omega$ is either slice or product, then $\mathcal{S R}(\Omega)$ is an integral domain or not. In the case when $\Omega$ is product, anyway, we have a partial zero-product property (for a proof see [16] or [18, Prop. 3.8]).

Proposition 2.8. If $\Omega$ is slice then $\mathcal{S} \mathcal{R}(\Omega)$ is an integral domain. If $\Omega$ is product, then $f \neq 0$ is a zero-divisor iff $f^{S}=0$. Moreover, if $\alpha \in S \mathcal{R}_{\mathbb{R}}(\Omega)$ and $f \in \mathcal{S R}(\Omega)$ are such that $\alpha f=0$, then either $\alpha=0$ or $f=0$.

We now introduce two operators on $\mathcal{S} \mathcal{R}(\Omega)$ which mime the behaviour of the scalar product on $\mathbb{H}$ and of the vector product on $\operatorname{Im} \mathbb{H} \simeq \mathbb{R}^{3}$.

Definition 2.9. Given $f, g \in \mathcal{S} \mathcal{R}(\Omega)$ we set

$$
\langle f, g\rangle_{\star}=\left(f^{\star} g^{c}\right)_{0} \in \mathcal{S} \mathcal{R}_{\mathbb{R}}(\Omega), \quad f_{\text {A }} g=\frac{f^{\star} g-g \star}{2} .
$$

The following proposition depicts the features of the operators $\langle,\rangle_{\star}$ and $A$ and their relation with the *product (for a proof and more details on the application of this result, see [2]).

Proposition 2.10. Let $f=f_{0}+f_{v}, g=g_{0}+g_{v} \in \mathcal{S} \mathcal{R}(\Omega)$. then

- $\langle f, g\rangle_{\star}=\langle g, f\rangle_{\star}=f_{0} g_{0}-\left(f_{v}{ }^{\star} g_{v}\right)_{0}$, 
- $f_{\AA} \mathbb{A} g=f_{V} \wedge g_{V}=-g \wedge A$,

- $f^{\star} g=f_{0} g_{0}-\left\langle f_{v}, g_{v}\right\rangle_{\star}+f_{0} g_{v}+g_{0} f_{v}+f_{v} \wedge g_{v}$.

We now define the main object of our study, the operators $\exp _{\star}, \cos _{\star}, \sin _{\star}, \cosh _{\star}$ and $\sinh _{\star}$ (the first three ones were introduced by Colombo, Sabadini and Struppa in [9]). The behaviour of exp ${ }_{\star}$ was studied in [2], while in [6] and in [10] the existence and uniqueness of the solution of equation $\exp _{\star}(f)=g$ was investigated, providing many results on the ${ }^{\star}$-logarithm of a slice regular function.

Definition 2.11. Given $f \in \mathcal{S} \mathcal{R}(\Omega)$, we set

$$
\begin{gathered}
\exp _{\star}(f)=\sum_{n \in \mathbb{N}} \frac{f^{\star} n}{n !}, \quad \cos *(f)=\sum_{n \in \mathbb{N}} \frac{(-1)^{n} f^{\star}(2 n)}{(2 n) !}, \quad \sin _{\star}(f)=\sum_{n \in \mathbb{N}} \frac{(-1)^{n} f^{\star}(2 n+1)}{(2 n+1) !}, \\
\cosh _{\star}(f)=\sum_{n \in \mathbb{N}} \frac{f^{\star(2 n)}}{(2 n) !}, \quad \sinh _{\star}(f)=\sum_{n \in \mathbb{N}} \frac{f^{\star}(2 n+1)}{(2 n+1) !} .
\end{gathered}
$$

Since all the power series involved in the definition have infinite radius of convergence, all these transcendental operators act on $\mathcal{S R}(\Omega)$.

Remark 2.12. For any $f \in \mathcal{S} \mathcal{R}(\Omega)$, a straightforward computation gives

$$
\begin{aligned}
\cosh _{\star}(f) & =\frac{\exp _{\star}(f)+\exp _{\star}(-f)}{2} \\
\sinh _{\star}(f) & =\frac{\exp _{\star}(f)-\exp _{\star}(-f)}{2} \\
\exp _{\star}(f) & =\cosh _{\star}(f)+\sinh _{\star}(f)
\end{aligned}
$$

The relations which link $\exp _{\star}, \cos \star$ and $\sin \star$ will be examined in Section 4 .

Remark 2.13. If $f \in \mathcal{S} \mathcal{R}_{\mathbb{R}}(\Omega)$, then $f^{\star}$ coincides with the pointwise product $f^{n}=f \cdots f$, thus $\exp _{\star}(f)=$ exp of, where exp denotes the exponential function exp : $\mathbb{H} \rightarrow \mathbb{H}$, and the same holds for all operators given above. Furthermore, if $f$ is $\mathbb{C}_{I}$ preserving for some $I \in \mathbb{S}$, then the restriction $\left(f^{\star n}\right)_{I}$ coincides with $f_{I} \cdots f_{I}=\left(f_{I}\right)^{n}$, so $\left(\exp _{\star}(f)\right)_{I}$ is equal to $\exp \circ f_{I}$, which entails $\exp _{\star}(f)=\operatorname{ext}\left(\exp \circ f_{I}\right)$, where ext denotes the regular extension of the holomorphic function $\exp \circ f_{I}: \Omega_{I} \rightarrow \mathbb{C}_{I}$ to the circular domain $\Omega$ (for a detailed description of the extension procedure, see [13], pp. 8-10). Again, the same relation holds for all operators introduced in Definition 2.11.

Thanks to the above remark, in order to simplify notations, when $f$ is slice preserving we often write $\exp (f)$ instead of $\exp _{\star}(f)$ and the same for $\cos *, \sin _{*}, \cosh \star$ and $\sinh _{\star}$.

Standard computations with power series (see e.g. [19]) show how the above operators act on the sum of two slice regular functions which commute under the ${ }^{\star}$-product.

Proposition 2.14. Let $f, g \in \mathcal{S} \mathcal{R}(\Omega)$ be such that $f^{\star} g=g$ * $f$. Then

$$
\begin{aligned}
& \exp _{\star}(f+g)=\exp _{\star}(f) \star \exp _{\star}(g) \\
& \cos \star(f+g)=\cos \star(f) \star \cos \star(g)-\sin \star(f) \star \sin _{\star}(g), \\
& \sin _{\star}(f+g)=\cos \star(f) \star \sin (g)+\sin (f){ }^{\star} \cos \star(g), \\
& \cosh _{\star}(f+g)=\cosh _{\star}(f){ }^{\star} \cosh _{\star}(g)+\sinh _{\star}(f){ }^{\star} \sinh \star(g), \\
& \sinh _{\star}(f+g)=\cosh _{\star}(f){ }^{\star} \sinh _{\star}(g)+\sinh _{\star}(f){ }^{\star} \cosh *(g) .
\end{aligned}
$$

The previous Proposition gives us the possibility to classify quaternions on which cos takes the same value. An analogous statement holds for the operator $\cos \star$ (see Remark 2.21). 
Proposition 2.15. Let $q, q^{\prime} \in \mathbb{H}$ and $n \in \mathbb{Z}$ be such that $\cos (q)=(-1)^{n} \cos \left(q^{\prime}\right)$. Then either $q$ and $q^{\prime}$ belong to the same slice and there exists $m \in \mathbb{Z}$ such that $m \equiv n$ (mod. 2) and $q^{\prime}= \pm q+m \pi$ or there exist $m, p \in \mathbb{Z}$ such that $m-p \equiv n$ (mod. 2), $q_{0}=m \pi, q_{0}^{\prime}=p \pi$ and $\left|q_{v}\right|=\left|q_{v}^{\prime}\right|$. In this last case, in particular we have $\cos (q), \cos \left(q^{\prime}\right) \in \mathbb{R}$.

Proof. If $q$ and $q^{\prime}$ belong to the same slice $\mathbb{C}_{I}$, then we can apply the properties of the function cosine on complex numbers and we are done. Otherwise write $q=q_{0}+q_{1} I$ and $q^{\prime}=q_{0}^{\prime}+q_{1}^{\prime} I^{\prime}$ for suitable $I, I^{\prime} \in$ $\mathbb{S}$ with $I \neq \pm I^{\prime}$ and $q_{1}, q_{1}^{\prime} \neq 0$. As cos is a slice-preserving function, then $\cos (q)=(-1)^{n} \cos \left(q^{\prime}\right)$ implies $\cos (q) \in \mathbb{R}$. Since $\cos (q)=\cos \left(q_{0}\right) \cos \left(q_{1} I\right)-\sin \left(q_{0}\right) \sin \left(q_{1} I\right)=\cos \left(q_{0}\right) \cosh \left(q_{1}\right)-\sin \left(q_{0}\right) \sinh \left(q_{1}\right) I$ and $\cos \left(q^{\prime}\right)=\cos \left(q_{0}^{\prime}\right) \cosh \left(q_{1}^{\prime}\right)-\sin \left(q_{0}^{\prime}\right) \sinh \left(q_{1}^{\prime}\right) I^{\prime}$, this gives $\sin \left(q_{0}\right) \sinh \left(q_{1}\right)=\sin \left(q_{0}^{\prime}\right) \sinh \left(q_{1}^{\prime}\right)=0$. The fact that sinh is never-vanishing on $\mathbb{R} \backslash\{0\}$ then entails that there exist $m, p \in \mathbb{Z}$ such that $q_{0}=m \pi$ and $q_{0}^{\prime}=p \pi$. We thus obtain $\cos (m \pi) \cosh \left(q_{1}\right)=(-1)^{n} \cos (p \pi) \cosh \left(q_{1}^{\prime}\right)$ which entails $m-p \equiv n(\bmod .2)$ and $q_{1}= \pm q_{1}^{\prime}$, and we are done.

In [2] and [6], the following features of $\exp _{\star}$ were proved.

Proposition 2.16. Let $f=f_{0}+f_{v}$. Then

- $\left(\exp _{\star}(f)\right)^{c}=\exp _{\star}\left(f^{c}\right)$,

- $\left(\exp _{\star}(f)\right)^{s}=\exp \left(2 f_{0}\right)$,

- $\exp _{\star}(f) \star \exp _{\star}(-f)=1$,

- $\exp _{\star}(\pi \mathcal{J})=-1$,

- $\exp _{\star}\left(f_{v}\right)=1+f_{v}$, if $f_{v}$ is a zero divisor.

Moreover, if $f$ is one-slice preserving and $f=f_{0}+f_{1} I$ for some $I \in \mathbb{S}$, then

$$
\exp _{\star}(f)=\exp \left(f_{0}\right)\left(\cos \left(f_{1}\right)+\sin \left(f_{1}\right) I\right)
$$

The existence of a *-logarithm of a one-slice preserving never vanishing function defined on a slice contractible domain (see [6] for a definition of slice contractible domains, a detailed study of the *-logarithm and the proof of the statement below) together with last equality, allow us to solve a cosine-sine problem on slice-contractible domains.

Proposition 2.17. Let $\Omega$ be a slice-contractible domain. Given $a_{0}, a_{1} \in \mathcal{S} \mathcal{R}_{\mathbb{R}}(\Omega)$ such that $a_{0}^{2}+a_{1}^{2} \equiv 1$, there exists $\gamma \in \mathcal{S R}_{\mathbb{R}}(\Omega)$ such that

$$
\left\{\begin{array}{l}
\cos \star(\gamma)=a_{0} \\
\sin *(\gamma)=a_{1}
\end{array}\right.
$$

Moreover,

- if $\Omega$ is a slice domain and $\tilde{\gamma} \in \mathcal{S R}(\Omega)$ is another solution of (2.10), then there exists $k \in \mathbb{Z}$ such that $\tilde{\gamma} \equiv \gamma+2 k \pi$;

- if $\Omega$ is a product domain and $\tilde{\gamma} \in S \mathcal{R}_{\mathbb{R}}(\Omega)$ is another solution of (2.10), then there exists $k \in \mathbb{Z}$ such that $\tilde{\gamma} \equiv \gamma+2 k \pi$.

The fact that slice preserving functions commute with any slice regular function allows us to apply Proposition 2.14 to the case when the two summands are the real part and the vector part of a given function, respectively. This provides an interesting rewriting of $\exp _{\star}, \cos \star, \sin _{\star}, \cosh \star$ and $\sinh _{\star}$; in order to obtain this new form, we need to define two slice preserving functions.

Definition 2.18. We denote by $\mu, v: \mathbb{H} \rightarrow \mathbb{H}$ the slice preserving functions given by

$$
\mu(q)=\sum_{n \in \mathbb{N}} \frac{(-1)^{n} q^{n}}{(2 n) !}, \quad v(q)=\sum_{n \in \mathbb{N}} \frac{(-1)^{n} q^{n}}{(2 n+1) !} .
$$

Trivially, for any $q \in \mathbb{H}$ we have

$$
\mu\left(q^{2}\right)=\cos (q), \quad q v\left(q^{2}\right)=\sin (q), \quad \mu\left(-q^{2}\right)=\cosh (q), \quad q v\left(-q^{2}\right)=\sinh (q) .
$$


As $\mu$ and $v$ are slice preserving, their squares coincide with $\mu^{\star} \mu$ and $v^{\star} v$ and for any $q \in \mathbb{H}$ we also have

$$
\mu^{2}(q)+q v^{2}(q)=1 .
$$

The relations given in Proposition 2.14 and the fact that $f_{v} \star f_{v}=-f_{v}^{s}$ entail the following equalities which give a new interpretation of the behaviour of the operators in terms of $\mu$ and $v$.

Proposition 2.19. Let $f=f_{0}+f_{v}$, then

(i) $\exp _{\star}(f)=\exp \left(f_{0}\right)\left(\mu\left(f_{v}^{S}\right)+v\left(f_{v}^{s}\right) f_{v}\right)$,

(ii) $\cos _{*}(f)=\cos \left(f_{0}\right) \mu\left(-f_{v}^{S}\right)-\sin \left(f_{0}\right) v\left(-f_{v}^{S}\right) f_{v}$,

(iii) $\sin _{*}(f)=\sin \left(f_{0}\right) \mu\left(-f_{v}^{S}\right)+\cos \left(f_{0}\right) v\left(-f_{v}^{S}\right) f_{v}$

(iv) $\cosh _{*}(f)=\cosh \left(f_{0}\right) \mu\left(f_{v}^{S}\right)+\sinh \left(f_{0}\right) v\left(f_{v}^{S}\right) f_{v}$,

(v) $\sinh _{*}(f)=\sinh \left(f_{0}\right) \mu\left(f_{v}^{s}\right)+\cosh \left(f_{0}\right) v\left(f_{v}^{s}\right) f_{v}$.

Proof. (i) is Proposition 4.5 in [2].

(ii) By Proposition 2.14 we have $\cos \star(f)=\cos \left(f_{0}\right) \cos \star\left(f_{v}\right)-\sin \left(f_{0}\right) \sin _{\star}\left(f_{v}\right)$. Now

$$
\cos _{\star}\left(f_{v}\right)=\sum_{n \in \mathbb{N}} \frac{(-1)^{n}\left(f_{v}\right)^{\star 2 n}}{(2 n) !}=\sum_{n \in \mathbb{N}} \frac{(-1)^{n}\left(\left(f_{v}\right)^{\star 2}\right)^{\star n}}{(2 n) !}=\sum_{n \in \mathbb{N}} \frac{(-1)^{n}\left(-f_{v}^{s}\right)^{\star n}}{(2 n) !}=\sum_{n \in \mathbb{N}} \frac{(-1)^{n}\left(-f_{v}^{s}\right)^{n}}{(2 n) !}=\mu\left(-f_{v}^{s}\right),
$$

where the second-to-last equality is a consequence of the fact that $-f_{v}^{s}$ is a slice preserving function and thus its $n$-th *-power coincides with its $n$-th power for the pointwise product. Analogously

$$
\sin _{\star}\left(f_{v}\right)=v\left(-f_{v}^{S}\right) f_{v}, \quad \cosh _{*}\left(f_{v}\right)=\mu\left(f_{v}^{S}\right), \quad \sinh _{\star}\left(f_{v}\right)=v\left(f_{v}^{S}\right) f_{v} .
$$

The proofs of (iii)-(v) also follows from Proposition 2.14 and the above relations.

A first consequence of the statement of Proposition 2.19 is the fact that the operators $\cos \star$ and $\sin \star$ behave as usual cosine and sine, in particular they satisfy an analogue the Pythagorean Trigonometric Identity. Indeed we have

Proposition 2.20. Let $f \in \mathcal{S} \mathcal{R}(\Omega)$, then

$$
(\cos \star(f))^{\star 2}+\left(\sin _{\star}(f)\right)^{\star 2} \equiv 1 .
$$

Proof. Thanks to Proposition 2.19 (ii) and (iii) we have

$$
\begin{aligned}
(\cos \star(f))^{\star 2}+(\sin *(f))^{\star 2} & =\left(\cos \left(f_{0}\right) \mu\left(-f_{v}^{s}\right)-\sin \left(f_{0}\right) v\left(-f_{v}^{s}\right) f_{v}\right)^{\star 2}+\left(\sin \left(f_{0}\right) \mu\left(-f_{v}^{s}\right)+\cos \left(f_{0}\right) v\left(-f_{v}^{s}\right) f_{v}\right)^{\star_{2}} \\
& =\cos ^{2}\left(f_{0}\right) \mu^{2}\left(-f_{v}^{s}\right)-2 \cos \left(f_{0}\right) \mu\left(-f_{v}^{s}\right) \sin \left(f_{0}\right) v\left(-f_{v}^{s}\right) f_{v}+\sin ^{2}\left(f_{0}\right) v^{2}\left(-f_{v}^{s}\right)\left(f_{v}\right)^{\star_{2}} \\
& +\sin ^{2}\left(f_{0}\right) \mu^{2}\left(-f_{v}^{s}\right)+2 \sin \left(f_{0}\right) \mu\left(-f_{v}^{s}\right) \cos \left(f_{0}\right) v\left(-f_{v}^{s}\right) f_{v}+\cos ^{2}\left(f_{0}\right) v^{2}\left(-f_{v}^{s}\right)\left(f_{v}\right)^{{ }_{2}} \\
& =\mu^{2}\left(-f_{v}^{s}\right)+v^{2}\left(-f_{v}^{s}\right)\left(-f_{v}^{s}\right) \equiv 1,
\end{aligned}
$$

where we applied the Pythagorean Trigonometric Identity for $\cos \left(f_{0}\right)$ and $\sin \left(f_{0}\right)$ and its analogue 2.12 and we used the equality $\left(f_{v}\right)^{\star 2}=-f_{v}^{S}$.

Proposition 2.19 also allows us to give a necessary condition on the functions in $\mathcal{S} \mathcal{R}(\Omega)$ on which the operator cos* takes the same value.

Remark 2.21. Let $f=f_{0}+f_{v}, g=g_{0}+g_{v} \in \mathcal{S} \mathcal{R}(\Omega)$ be such that

$$
\cos \star(f)=\cos \star(g) .
$$

Then either $f_{v}$ and $g_{v}$ are linearly dependent on $\mathcal{S} \mathcal{R}_{\mathbb{R}}(\Omega)$ or $\sin \left(f_{0}\right) v\left(-f_{v}^{S}\right)=\sin \left(g_{0}\right) v\left(-g_{v}^{S}\right) \equiv 0$. 
Proof. As $\cos *(f)=\cos \left(f_{0}\right) \mu\left(-f_{v}^{S}\right)-\sin \left(f_{0}\right) v\left(-f_{v}^{S}\right) f_{v}$ and $\cos *(g)=\cos \left(g_{0}\right) \mu\left(-g_{v}^{S}\right)-\sin \left(g_{0}\right) v\left(-g_{v}^{S}\right) g_{v}$, Equality 2.14 implies

$$
\sin \left(f_{0}\right) v\left(-f_{v}^{s}\right) f_{v}=\sin \left(g_{0}\right) v\left(-g_{v}^{S}\right) g_{v} .
$$

Then either $f_{v}$ and $g_{v}$ are linearly dependent on $\mathcal{S} \mathcal{R}_{\mathbb{R}}(\Omega)$ or $\sin \left(f_{0}\right) v\left(-f_{v}^{S}\right)=\sin \left(g_{0}\right) v\left(-g_{v}^{S}\right) \equiv 0$.

\section{The behaviour of $\mu$ and $v$}

A first study of the behaviour of the functions $\mu$ and $v$ was carried out in [6] and [10], focusing in particular on the identification of a fundamental domain for the map $\mu$ in the complex plane.

Here we deepen the analysis of the features of the functions $\mu$ and $v$ which will be applied in the next section to obtain better comprehension of the relations between $\cos \star$ and $\sin \star$ and their hyperbolic analogues $\cosh \star$ and $\sinh _{\star}$. A first couple of results are simple observations on the behaviour of $\mu$ and $\nu$ on the reals.

Remark 3.1. Let $x \in \mathbb{R}$, then $|\mu(x)| \leq 1$ if $x \geq 0$ and $\mu(x)>1$ if $x<0$. Indeed, if $x \geq 0$, then there exists $y \in \mathbb{R}$ such that $x=y^{2}$ and thus $|\mu(x)|=\left|\mu\left(y^{2}\right)\right|=|\cos (y)| \leq 1$. If $x<0$, then there exists $y \in \mathbb{R} \backslash\{0\}$ such that $x=-y^{2}$, so that $\mu(x)=\mu\left(-y^{2}\right)=\cosh (y)>1$.

Remark 3.2. The zero set of the function $v$ is $\left\{n^{2} \pi^{2}: n \in \mathbb{N}, n \neq 0\right\}$. First of all notice that $v(0)=1$, thus if $q \in \mathbb{H}$ is a zero of $v$, then $q \neq 0$. Now take $\xi \in \mathbb{H}$ such that $\xi^{2}=q$; as $0=\xi v(q)=\xi v\left(\xi^{2}\right)=\sin (\xi)$ and $\xi \neq 0$ we obtain $\xi=n \pi$ for a suitable $n \in \mathbb{N} \backslash\{0\}$. Thus $q=n^{2} \pi^{2}$ with $n \in \mathbb{N} \backslash\{0\}$. In particular, $v$ is never-vanishing on $(-\infty, 0]$.

Next proposition describes in detail when the function $\mu$ takes the same value, up to a change of sign, in two different points which differ by a real summand or which sum up to a real summand.

Proposition 3.3. (i) Let $n \in \mathbb{Z}, q \in \mathbb{H}$ and $c \in \mathbb{R} \backslash\{0\}$. Then

$$
\mu(q)=(-1)^{n} \mu(q+c) .
$$

if and only if there exists $p \in \mathbb{Z} \backslash\{0\}$ such that $p \equiv n$ (mod. 2) and $q=\left(\frac{p^{2} \pi^{2}-c}{2 p \pi}\right)^{2}$. In particular, if Equality 3.1 holds, then $q$ is real and non negative.

(ii) Let $n \in \mathbb{Z}, q \in \mathbb{H}$ and $c \in \mathbb{R}$. Then

$$
\mu(q)=(-1)^{n} \mu(-q+c) .
$$

if and only if there exists $p \in \mathbb{Z}$ such that $p \equiv n(\bmod .2)$ and $q=\frac{1}{2}\left(c \pm p \pi \sqrt{2 c-p^{2} \pi^{2}}\right)$ if $2 c-p^{2} \pi^{2} \geq 0$, while there exists $I \in \mathbb{S}$ such that $q=\frac{1}{2}\left(c+I p \pi \sqrt{p^{2} \pi^{2}-2 c}\right)$ if $2 c-p^{2} \pi^{2}<0$.

Proof. (i) Choose $\xi, \eta \in \mathbb{H}$ which belong to the same slice and such that $\xi^{2}=q$ and $\eta^{2}=q+c$ (this choice is always possible since $c \in \mathbb{R}$, so that $q$ and $q+c$ belong to the same slice as well). Equality 3.1 is therefore equivalent to $\mu\left(\xi^{2}\right)=(-1)^{n} \mu\left(\eta^{2}\right)$ which can also be written as $\cos (\xi)=(-1)^{n} \cos (\eta)$. Thanks to Proposition 2.15, last equality holds if and only if there exists $p \in \mathbb{Z}$ which has the same parity as $n$ and such that $\eta= \pm \xi+p \pi$. By taking the square on both sides we get

$$
q+c=\eta^{2}=( \pm \xi+p \pi)^{2}=\xi^{2} \pm 2 p \pi \xi+p^{2} \pi^{2}=q \pm 2 p \pi \xi+p^{2} \pi^{2} .
$$

Up to changing $p$ with $-p$ if necessary, this gives $\xi=\frac{p^{2} \pi^{2}-c}{2 p \pi}$ and thus $q=\left(\frac{p^{2} \pi^{2}-c}{2 p \pi}\right)^{2} \geq 0$.

(ii) Again, choose $\xi, \eta \in \mathbb{H}$ which belong to the same slice and such that $\xi^{2}=q$ and $\eta^{2}=-q+c$, that is $\xi^{2}+\eta^{2}=c$. By reasoning as above, Equality 3.2 holds if and only if there exists $p \in \mathbb{Z}$ with $p \equiv n$ (mod. 2) such that $\eta= \pm \xi+p \pi$. Up to changing $p$ with $-p$ if necessary, we get $2 \xi^{2}-2 p \pi \xi+p^{2} \pi^{2}-c=0$. Since all coefficients of this equation are real numbers, by trivial computations we obtain that if $2 c-p^{2} \pi^{2} \geq 0$, 
then $q=\left(\frac{p \pi \pm \sqrt{2 c-p^{2} \pi^{2}}}{2}\right)^{2}=\frac{1}{2}\left(c \pm p \pi \sqrt{2 c-p^{2} \pi^{2}}\right) \geq 0$, while if $2 c-p^{2} \pi^{2}<0$ there exists $I \in \mathbb{S}$ such that $q=\left(\frac{p \pi+I \sqrt{p^{2} \pi^{2}-2 c}}{2}\right)^{2}=\frac{1}{2}\left(c+I p \pi \sqrt{p^{2} \pi^{2}-2 c}\right)$.

An accurate analysis of the behaviour of $\mu$ and $v$ allows us to give a more refined statement for the solutions of the sine-cosine problem which is solved in [6, Proposition 4.6] and here is recalled as Proposition 2.17. As a matter of fact, its statement contains a general "uniqueness" result, up to multiples of $2 \pi$, for slice domains, while in the case of product domains the substantial "uniqueness" of the solution holds for slice preserving functions only (we recall that when the domain is slice-contractible the existence of solutions is guaranteed).

Proposition 3.4. Let $\Omega$ be a product domain and $a_{0}, a_{1} \in \mathcal{S} \mathcal{R}_{\mathbb{R}}(\Omega)$ be such that $a_{0}^{2}+a_{1}^{2} \equiv 1$. Suppose $\tilde{\gamma}_{0} \in$ $\mathcal{S R}_{\mathbb{R}}(\Omega)$ is a solution of the sine-cosine problem

$$
\left\{\begin{array}{l}
\cos \star(\gamma)=a_{0}, \\
\sin _{\star}(\gamma)=a_{1} .
\end{array}\right.
$$

If $\gamma=\gamma_{0}+\gamma_{v} \in \mathcal{S R}(\Omega)$ is another solution of System 3.3, then either $\gamma_{v} \equiv 0$ and there exists $n \in \mathbb{Z}$ such that $\gamma_{0}=\tilde{\gamma}_{0}+2 n \pi$ or there exist $m \in \mathbb{Z} \backslash\{0\}$ such that $\gamma_{v}^{s}=-m^{2} \pi^{2}$ and $n \in \mathbb{Z}$ such that $n \equiv m$ (mod.2) and $\gamma_{0}=\tilde{\gamma}_{0}+n \pi$.

Proof. Thanks to Proposition 2.19 cases (ii) and (iii), we have that System 3.3 is equivalent to

$$
\left\{\begin{array}{l}
\cos \left(\gamma_{0}\right) \mu\left(-\gamma_{v}^{s}\right)-\sin \left(\gamma_{0}\right) v\left(-\gamma_{v}^{s}\right) \gamma_{v}=a_{0}, \\
\sin \left(\gamma_{0}\right) \mu\left(-\gamma_{v}^{s}\right)+\cos \left(\gamma_{0}\right) v\left(-\gamma_{v}^{s}\right) \gamma_{v}=a_{1} .
\end{array}\right.
$$

Since both $a_{0}$ and $a_{1}$ are slice preserving, we obtain $\sin \left(\gamma_{0}\right) v\left(-\gamma_{v}^{s}\right) \gamma_{v}=\cos \left(\gamma_{0}\right) v\left(-\gamma_{v}^{s}\right) \gamma_{v} \equiv 0$. If $\gamma_{v} \equiv 0$, then $\gamma$ belongs to $S \mathcal{R}_{\mathbb{R}}(\Omega)$ and the "uniqueness" proved in [6, Proposition 4.6] yields the conclusion (notice that the part of the proof concerning the "uniqueness" does not depend on the fact that the domain is slicecontractible).

If $\gamma_{v} \not \equiv 0$, then $\sin \left(\gamma_{0}\right) v\left(-\gamma_{v}^{S}\right)=\cos \left(\gamma_{0}\right) v\left(-\gamma_{v}^{S}\right) \equiv 0$. By taking the square of both terms and summing up, thanks to $\cos \left(\gamma_{0}\right)^{2}+\sin \left(\gamma_{0}\right)^{2} \equiv 1$, we obtain $v\left(-\gamma_{v}^{s}\right)^{2} \equiv 0$ and thus $v\left(-\gamma_{v}^{s}\right) \equiv 0$. By Remark 3.2 we therefore find $m \in \mathbb{Z} \backslash\{0\}$ such that $\gamma_{v}^{s}=-m^{2} \pi^{2}$. Moreover, since $\mu\left(-\gamma_{v}^{s}\right)=\mu\left(m^{2} \pi^{2}\right)=(-1)^{m}$, if $m$ is even we have

$$
\left\{\begin{array}{l}
\cos \left(\gamma_{0}\right)=a_{0}, \\
\sin \left(\gamma_{0}\right)=a_{1},
\end{array}\right.
$$

which entails $\gamma_{0}=\tilde{\gamma}_{0}+n \pi$ for a suitable $n \in \mathbb{Z}$ even, while if $m$ is odd we obtain

$$
\left\{\begin{array}{l}
\cos \left(\gamma_{0}\right)=-a_{0}, \\
\sin \left(\gamma_{0}\right)=-a_{1},
\end{array}\right.
$$

and thus

$$
\left\{\begin{array}{l}
\cos \left(\gamma_{0}+\pi\right)=a_{0}, \\
\sin \left(\gamma_{0}+\pi\right)=a_{1},
\end{array}\right.
$$

which implies that $\gamma_{0}=\tilde{\gamma}_{0}+n \pi$ for a suitable $n \in \mathbb{Z}$ odd.

\section{Relations between trigonometric operators and their hyperbolic counterparts}

In Remark 2.12 we saw that the operators $\exp _{\star}, \cosh \star$ and $\sinh \star$ are related by the same equalities that hold on domains contained in $\mathbb{C}$. Nevertheless, when we deal with $\cos \star$ and $\sin \star$, a straightforward generalization of the usual relations which hold on complex numbers is not always possible. 
The existence of the function $\mathcal{J}$ introduced in Definition 2.3 makes the case of product domains analogous to the complex case, where $\cos (z)=\cosh (\imath z)$ and $\sin (z)=\frac{\sinh (l z)}{l}$ for any $z \in \mathbb{C}$, and $\imath$ denotes the imaginary unit in $\mathbb{C}$.

Proposition 4.1. Let $\Omega$ be a product domain. Then for any $f \in \mathcal{S} \mathcal{R}(\Omega)$ we have

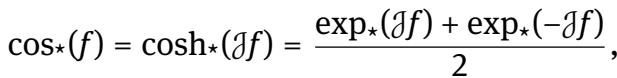

$$
\begin{aligned}
& \sin _{*}(f)=\frac{\sinh _{*}(\mathcal{J} f)}{\mathcal{J}}=\frac{\exp _{\star}(\partial f)-\exp _{*}(-\partial f)}{2 \mathcal{J}} .
\end{aligned}
$$

Proof. Indeed

$$
\cosh *(\partial f)=\sum_{n \in \mathbb{N}} \frac{(\partial f)^{\star(2 n)}}{(2 n) !}=\sum_{n \in \mathbb{N}} \frac{\partial^{2 n} f^{\star(2 n)}}{(2 n) !}=\sum_{n \in \mathbb{N}} \frac{(-1)^{n} f^{\star(2 n)}}{(2 n) !}=\cos \star(f),
$$

where the second equality is due to the fact that $\mathcal{J}$ is slice preserving and thus stays in the center of $\mathcal{S} \mathcal{R}(\Omega)$ and the third equality holds because $\partial^{2}=-1$. Analogously

$$
\sinh _{*}(\mathcal{J} f)=\sum_{n \in \mathbb{N}} \frac{(\mathcal{J} f)^{\star(2 n+1)}}{(2 n+1) !}=\sum_{n \in \mathbb{N}} \frac{\partial \mathcal{J}^{2 n} f^{\star(2 n+1)}}{(2 n+1) !}=\sum_{n \in \mathbb{N}} \frac{\mathcal{J}(-1)^{n} f^{\star(2 n+1)}}{(2 n+1) !}=\mathcal{J} \sin _{*}(f) .
$$

Notice that Equality 4.2 makes sense because the function $\mathcal{J}$ belongs to $\mathcal{S} \mathcal{R}_{\mathbb{R}}(\Omega)$ and is never-vanishing, thus the quotient $\frac{\sinh *(\partial f)}{\partial}$ is well defined.

Next result shows that the usual relation between ${ }^{\star}$-cosine and hyperbolic ${ }^{\star}$-cosine (as well as the one between ${ }^{\star}$-sine and hyperbolic *-sine) holds for one-slice preserving functions defined on a domain $\Omega$, both slice or product.

Proposition 4.2. Let $I \in \mathbb{S}$ and $f \in \mathcal{S R}_{I}(\Omega)$. Then

$$
\begin{aligned}
& \cos _{\star}(f)=\cosh _{\star}(f \star I)=\frac{\exp _{\star}\left(f^{\star} I\right)+\exp _{\star}(-f \star I)}{2}, \\
& \sin _{\star}(f)=\sinh _{\star}\left(f^{\star} I\right) \star I^{-\star}=\left(\exp _{\star}\left(f^{\star} I\right)-\exp _{\star}\left(-f^{\star} I\right)\right) \star(2 I)^{-\star} .
\end{aligned}
$$

Proof. In both lines the second equality is an immediate consequence of the first one and of Remark 2.12.

Let us consider the restriction $f_{I}$ of $f$ to $\Omega_{I}$ : since $f$ is $\mathbb{C}_{I}$-preserving, then $f{ }^{\star} I=f I$ is again $\mathbb{C}_{I}$-preserving and thus $\left((f \star I)^{\star} n\right)_{I}=\left((f \star I)_{I}\right)^{n}=\left(f_{I}\right)^{n} \star I^{n}$, where last equality is due to the fact that $f_{I}$ and $I$ commute, since they both take value in $\mathbb{C}_{I}$.

By considering the restriction of $\cosh _{\star}\left(f^{\star} I\right)$ to $\mathbb{C}_{I}$ we therefore obtain

$$
\left(\cosh _{\star}\left(f^{\star} I\right)\right)_{I}=\sum_{n \in \mathbb{N}} \frac{\left((f \star I)^{\star(2 n)}\right)_{I}}{(2 n) !}=\sum_{n \in \mathbb{N}} \frac{\left(f_{I}\right)^{2 n} I^{2 n}}{(2 n) !}=\sum_{n \in \mathbb{N}}(-1)^{n} \frac{\left(f_{I}\right)^{\star(2 n)}}{(2 n) !}=(\cos \star(f))_{I} .
$$

The Identity Principle then entails $\cos \star(f)=\cosh \star(f \star I)$.

The first equality of 4.4 is equivalent to $\sin \star(f) \star I=\sinh \star(f \star I)$. By considering the restriction of the function $\sinh \star(f \star I)$ to $\Omega_{I}$, we obtain by following the same lines of reasoning as above

$$
(\sinh \star(f \star I))_{I}=\sum_{n \in \mathbb{N}}(-1)^{n} \frac{\left(f_{I}\right)^{\star(2 n+1)}}{(2 n+1) !} I=(\sin \star(f))_{I} I .
$$

As the functions $\sin \star(f)$ and $I$ are both $\mathbb{C}_{I}$-preserving, then by Remark 2.5 we have $(\sinh \star(f \star I))_{I}=\left(\sin _{\star}(f) \star I\right)_{I}$ and thus another application of the Identity Principle gives $\sinh \star(f \star I)=\sin _{\star}(f) \star I$, which concludes the proof.

Remark 4.3. Notice that the ${ }^{\star}$-product $\sinh \star(f \star I){ }^{\star} I^{-\star}$ coincides with the pointwise product $-\sinh \star(f \star I) I$, since the second factor $I^{-^{\star}}=-I$ is constant. 
A natural question follows from the above result: if Equality 4.3 (or 4.4) holds for $I \in \mathbb{S}$ and $f \in \mathcal{S} \mathcal{R}(\Omega)$, does this imply that $f$ is $\mathbb{C}_{I}$-preserving? When the domain $\Omega$ is slice, the answer is positive, as shown by the following

Theorem 4.4. Let $\Omega$ be a slice domain. If $I \in \mathbb{S}$ and $f \in \mathcal{S} \mathcal{R}(\Omega)$ are such that

$$
\cos \star(f)=\cosh \star(f \star I),
$$

then $f$ is $\mathbb{C}_{I}$-preserving.

Proof. Complete 1 and $I$ to an orthonormal basis 1, $I, J, K$ of $\mathbb{H}$ and write $f=f_{0}+f_{1} I+f_{2} J+f_{3} K=f_{0}+f_{v}$.

By Proposition 2.19 (ii) and (iv) we have

$$
\cos _{\star}(f)=\cos \left(f_{0}\right) \mu\left(-f_{v}^{s}\right)-\sin \left(f_{0}\right) v\left(-f_{v}^{s}\right) f_{v}
$$

and, on the other hand,

$$
\begin{aligned}
\cosh _{\star}\left(f^{\star} I\right) & =\cosh \star\left(-f_{1}+f_{0} I+f_{3} J-f_{2} K\right) \\
& =\cosh \left(-f_{1}\right) \mu\left(f_{0}^{2}+f_{3}^{2}+f_{2}^{2}\right)+\sinh \left(-f_{1}\right) v\left(f_{0}^{2}+f_{3}^{2}+f_{2}^{2}\right)\left(f_{0} I+f_{3} J-f_{2} K\right) .
\end{aligned}
$$

Since $\cosh \left(-f_{1}\right)=\cosh \left(f_{1}\right)$ and $\sinh \left(-f_{1}\right)=-\sinh \left(f_{1}\right)$, by the uniqueness statement of Proposition 2.6, we can write Equality 4.5 in the form

$$
\left\{\begin{array}{l}
\cosh \left(f_{1}\right) \mu\left(f_{0}^{2}+f_{3}^{2}+f_{2}^{2}\right)=\cos \left(f_{0}\right) \mu\left(-f_{1}^{2}-f_{2}^{2}-f_{3}^{2}\right) \\
\sinh \left(f_{1}\right) v\left(f_{0}^{2}+f_{3}^{2}+f_{2}^{2}\right) f_{0}=\sin \left(f_{0}\right) v\left(-f_{v}^{s}\right) f_{1} \\
\sinh \left(f_{1}\right) v\left(f_{0}^{2}+f_{3}^{2}+f_{2}^{2}\right) f_{3}=\sin \left(f_{0}\right) v\left(-f_{v}^{s}\right) f_{2} \\
-\sinh \left(f_{1}\right) v\left(f_{0}^{2}+f_{3}^{2}+f_{2}^{2}\right) f_{2}=\sin \left(f_{0}\right) v\left(-f_{v}^{s}\right) f_{3}
\end{array}\right.
$$

Let us consider the homogeneous system arising from the two last equations

$$
\left\{\begin{array}{l}
\sin \left(f_{0}\right) v\left(-f_{v}^{s}\right) f_{2}-\sinh \left(f_{1}\right) v\left(f_{0}^{2}+f_{3}^{2}+f_{2}^{2}\right) f_{3}=0 \\
\sinh \left(f_{1}\right) v\left(f_{0}^{2}+f_{3}^{2}+f_{2}^{2}\right) f_{2}+\sin \left(f_{0}\right) v\left(-f_{v}^{s}\right) f_{3}=0 .
\end{array}\right.
$$

We then have that either $f_{2}=f_{3} \equiv 0$, which means that $f=f_{0}+f_{1} I$, i.e. that $f$ is $\mathbb{C}_{I}$-preserving, and we are done, or

$$
\left(\sin \left(f_{0}\right) v\left(-f_{v}^{s}\right)\right)^{2}+\left(\sinh \left(f_{1}\right) v\left(f_{0}^{2}+f_{3}^{2}+f_{2}^{2}\right)\right)^{2} \equiv 0 .
$$

Since $f_{0},-f_{v}^{s}, f_{1}$ and $f_{0}^{2}+f_{3}^{2}+f_{2}^{2}$ are all slice-preserving, and the same holds for $v$, sin and sinh, then the functions $\sin \left(f_{0}\right), v\left(-f_{v}^{S}\right), \sinh \left(f_{1}\right)$ and $v\left(f_{0}^{2}+f_{3}^{2}+f_{2}^{2}\right)$ which appear in Equation 4.8 take real values on $\Omega \cap \mathbb{R}$. Thus their squares are non-negative and hence we have

$$
\left\{\begin{array}{l}
\sin \left(f_{0}\right) v\left(-f_{v}^{s}\right)=0 \\
\sinh \left(f_{1}\right) v\left(f_{0}^{2}+f_{3}^{2}+f_{2}^{2}\right)=0
\end{array}\right.
$$

on $\Omega \cap \mathbb{R}$ and thus on $\Omega$ by the Identity Principle. Let us start by considering the first equation of System 4.9: as the domain $\Omega$ is slice, then $\mathcal{S R}(\Omega)$ is an integral domain and thus either $v\left(-f_{v}^{S}\right) \equiv 0$ or $\sin \left(f_{0}\right) \equiv 0$. As $-f_{v}^{s}$ takes non positive values on $\Omega \cap \mathbb{R}$, by Remark 3.2 we have that $v\left(-f_{v}^{s}\right)$ cannot vanish on $\Omega \cap \mathbb{R}$ and thus $\sin \left(f_{0}\right) \equiv 0$.

Then there exists $n \in \mathbb{Z}$ such that $f_{0}=n \pi$; by substituting in the second equation we get either $\sinh \left(f_{1}\right) \equiv$ 0 or $v\left(n^{2} \pi^{2}+f_{2}^{2}+f_{3}^{2}\right) \equiv 0$. Since $f_{1}$ is slice-preserving and $\Omega$ is slice, first equation is equivalent to $f_{1} \equiv 0$; in this case the first equation of System 4.6 becomes

$$
\mu\left(n^{2} \pi^{2}+f_{3}^{2}+f_{2}^{2}\right)=(-1)^{n} \mu\left(-f_{2}^{2}-f_{3}^{2}\right) .
$$


By Remark 3.1, since $n^{2} \pi^{2}+f_{3}^{2}+f_{2}^{2} \geq 0$ on $\Omega \cap \mathbb{R}$, we have $\left|\mu\left(n^{2} \pi^{2}+f_{3}^{2}+f_{2}^{2}\right)\right| \leq 1$, while $-f_{2}^{2}-f_{3}^{2} \leq 0$ on $\Omega \cap \mathbb{R}$ gives $\left|(-1)^{n} \mu\left(-f_{2}^{2}-f_{3}^{2}\right)\right| \geq 1$. Thus the only possibility in which equality holds is when $f_{2}^{2}+f_{3}^{2}=0$ on $\Omega \cap \mathbb{R}$. This implies $f_{2}=f_{3}=0$ on $\Omega \cap \mathbb{R}$ and the Identity Principle entails again $f_{2}=f_{3} \equiv 0$, that is $f$ is $\mathbb{C}_{I}$-preserving.

We now examine when $v\left(n^{2} \pi^{2}+f_{2}^{2}+f_{3}^{2}\right) \equiv 0$. In this case by Remark 3.2 we find $m \in \mathbb{Z} \backslash\{0\}$ such that $n^{2} \pi^{2}+f_{2}^{2}+f_{3}^{2}=m^{2} \pi^{2}$ and therefore $f_{2}^{2}+f_{3}^{2}=\left(m^{2}-n^{2}\right) \pi^{2}$. Thus the first equation of System 4.6 can be writen as $(-1)^{m} \cosh \left(f_{1}\right)=(-1)^{n} \mu\left(-f_{1}^{2}+\left(n^{2}-m^{2}\right) \pi^{2}\right)$ or equivalently

$$
\mu\left(-f_{1}^{2}\right)=(-1)^{n-m} \mu\left(-f_{1}^{2}+\left(n^{2}-m^{2}\right) \pi^{2}\right) .
$$

On $\Omega \cap \mathbb{R}$ the function $-f_{1}^{2}$ takes negative values, and thus we can apply Proposition 3.3 case (i) and obtain $n^{2}-m^{2}=0$. Since $f_{2}^{2}+f_{3}^{2}=\left(m^{2}-n^{2}\right) \pi^{2}=0$, we have $f_{2}=f_{3}=0$ on $\Omega \cap \mathbb{R}$ and thus $f_{2}=f_{3} \equiv 0$ on $\Omega$ again by the Identity Principle, which anew implies that $f$ is $\mathbb{C}_{I}$-preserving.

When we move to a product domain the above statement is not valid anymore and there exists a large class of slice regular functions which are not $\mathbb{C}_{I}$-preserving such that Equality 4.5 holds.

In the statement of the following result, for points (iii)-(vi) we extend $1, I$ to any orthonormal basis $1, I, J, K$ of $\mathbb{H}$ and write $f=f_{0}+f_{1} I+f_{2} J+f_{3} K$. Indeed, notice that the condition on the last two components $f_{2}$ and $f_{3}$ is always stated in terms of $f_{2}^{2}+f_{3}^{2}$, so it is independent from any choice of the extension, since $J, K$ is an orthonormal basis of the orthogonal complement of $\mathbb{R} \oplus \mathbb{R} I$ in $\mathbb{H}$.

Theorem 4.5. Let $\Omega$ be a product domain, $I \in \mathbb{S}$ and $f \in \mathcal{S} \mathcal{R}(\Omega)$. Then

$$
\cos _{*}(f)=\cosh _{*}(f \star I)
$$

is satisfied if and only if one of the following conditions holds

(i) $f$ is $\mathbb{C}_{I}$-preserving;

(ii) there exist $J \in \mathbb{S}$ orthogonal to I and $f_{0}, f_{2} \in \mathcal{S} \mathcal{R}_{\mathbb{R}}(\Omega)$ such that $f=2\left(f_{0}+f_{2} J\right) \star \ell_{I}^{ \pm}$, where $\ell_{I}^{ \pm}$is defined in 2.3;

(iii) there exist $n, m, p \in \mathbb{Z}$ such that $p \equiv n-m(\bmod .2), f_{0} \equiv n \pi, f_{1} \equiv m \mathcal{J} \pi$ and

$$
f_{2}^{2}+f_{3}^{2}=\left\{\begin{array}{l}
-\frac{\pi^{2}}{2}\left[\left(n^{2}+m^{2}\right) \pm p \sqrt{2 n^{2}-2 m^{2}-p^{2}}\right], \text { if } 2 n^{2}-2 m^{2}-p^{2} \geq 0, \\
-\frac{\pi^{2}}{2}\left[\left(n^{2}+m^{2}\right) \pm J p \sqrt{2 m^{2}-2 n^{2}+p^{2}}\right] \text { if } 2 n^{2}-2 m^{2}-p^{2}<0 ;
\end{array}\right.
$$

(iv) there exist $n, m, p \in \mathbb{Z}$ with $m, p \neq 0$ such that $p \equiv n-m$ (mod. 2$), f_{0} \equiv n \pi, f_{1}=J \pi \frac{p^{2}+m^{2}-n^{2}}{2 p}$ and $f_{2}^{2}+f_{3}^{2}=\left(m^{2}-n^{2}\right) \pi^{2}$

(v) there exist $n, m, p \in \mathbb{Z}$ with $m, p \neq 0$ and such that $p \equiv n-m$ (mod.2), $f_{0}=\frac{p^{2}+n^{2}-m^{2}}{2 p} \pi, f_{1} \equiv n \mathcal{J} \pi$ and $f_{2}^{2}+f_{3}^{2}=\left(m^{2}-n^{2}\right) \pi^{2}$

(vi) there exist $n, m, p \in \mathbb{Z}$ with $n, m \neq 0$ and $p \equiv n-m$ (mod. 2) such that $f_{0}=\frac{p \pm \sqrt{2\left(n^{2}+m^{2}\right)-p^{2}}}{2} \pi, f_{1}=$ $\mathrm{g} \frac{p \mp \sqrt{2\left(n^{2}+m^{2}\right)-p^{2}}}{2} \pi$ and $f_{2}^{2}+f_{3}^{2}=\frac{1}{2}\left[\left(n^{2}-m^{2}\right) \mp p \sqrt{2\left(n^{2}+m^{2}\right)-p^{2}}\right] \pi^{2}$ if $2\left(n^{2}+m^{2}\right)-p^{2} \geq 0$, while if $2\left(n^{2}+m^{2}\right)-p^{2}<0$, then we have $f_{0}=\frac{p \pm \mathcal{J} \sqrt{p^{2}-2\left(n^{2}+m^{2}\right)}}{2} \pi, f_{1}=\mathcal{J} \frac{p \mp \mathcal{J} \sqrt{p^{2}-2\left(n^{2}+m^{2}\right)}}{2} \pi$ and $f_{2}^{2}+f_{3}^{2}=$ $\frac{1}{2}\left[\left(n^{2}-m^{2}\right) \mp J p \sqrt{p^{2}-2\left(n^{2}+m^{2}\right)}\right] \pi^{2}$.

Proof. First of all notice that, since $f^{\star} I=\mathcal{J}(-\mathcal{J} f \star I)$ and $\cos \star$ is even, by Proposition 4.1 we can write 4.10 in the form

$$
\cos \star(f)=\cos \star(J f \star I)
$$

Proposition 4.2 shows that Equality 4.10 holds in case (i), while the sufficiency of case (ii) is obtained by direct inspection, since when $f=2\left(f_{0}+\partial f_{2}\right) \star \ell_{I}^{ \pm}$we have $\partial f \star I=-f$. In all cases (iii)-(vi) a direct inspection gives $\cos \star(f)=\cos \star(J f \star I)$.

Now suppose that Equality 4.11 holds. Thanks to Remark 2.21 and the fact that

$$
\mathcal{\partial} f^{\star} I=-\partial f_{1}+\partial f_{0} I+\partial f_{3} J-\partial f_{2} K,
$$


we obtain that either the functions $f_{v}$ and $\left(\delta f^{\star} I\right)_{v}$ are linearly dependent on $\delta \mathcal{R}_{\mathbb{R}}(\Omega)$ or we have $\sin \left(f_{0}\right) v\left(-f_{v}^{S}\right)=$ $\sin \left(\partial f_{1}\right) v\left(f_{0}^{2}+f_{3}^{2}+f_{2}^{2}\right) \equiv 0$.

Let us consider first when $f_{v}$ and $(f f \star I)_{v}$ are linearly dependent on $\mathcal{S} \mathcal{R}_{\mathbb{R}}(\Omega)$. In this case there exist $\alpha, \beta \in \mathcal{S} \mathcal{R}_{\mathbb{R}}(\Omega)$ not both identically zero such that $\alpha f_{v}=\beta(\mathcal{J} f \star I)_{v}$. This equality is equivalent to

$$
\left\{\begin{array}{l}
\alpha f_{1}=\beta \partial f_{0}, \\
\alpha f_{2}=\beta \partial f_{3}, \\
\alpha f_{3}=-\beta J f_{2} .
\end{array}\right.
$$

If $f$ is not $\mathbb{C}_{I}$-preserving, then the homogeneous linear system consisting of the last two equations must have zero determinant, so that $\alpha^{2}-\beta^{2} \equiv 0$. As $\delta \mathcal{R}_{\mathbb{R}}(\Omega)$ is an integral domain, then either $\alpha \equiv \beta$ or $\alpha \equiv-\beta$. In the first case, $f_{1}=\mathcal{J} f_{0}, f_{3}=-\partial f_{2}$ and thus

$$
f=f_{0}+\partial f_{0} I+f_{2} J-\partial f_{2} K=f_{0}(1+\partial I)+f_{2} J(1+\partial I)=2\left(f_{0}+f_{2} J\right) \star \ell_{I}^{-} .
$$

Analogously, if $\alpha=-\beta$ we find $f=2\left(f_{0}+f_{2} J\right) \star \ell_{I}^{+}$.

Now we are left to consider the case when $f_{v}$ and $\left(f f^{\star} I\right)_{v}$ are linearly independent on $\mathcal{S} \mathcal{R}_{\mathbb{R}}(\Omega)$ which gives $\sin \left(f_{0}\right) v\left(-f_{v}^{S}\right)=\sin \left(J f_{1}\right) v\left(f_{0}^{2}+f_{3}^{2}+f_{2}^{2}\right) \equiv 0$.

Since $\delta \mathcal{R}_{\mathbb{R}}(\Omega)$ is an integral domain this implies that we have to deal with four different possibilities

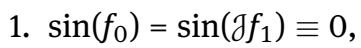

2. $\sin \left(f_{0}\right)=v\left(f_{0}^{2}+f_{3}^{2}+f_{2}^{2}\right) \equiv 0$,

3. $v\left(-f_{v}^{S}\right)=\sin \left(\mathcal{J} f_{1}\right) \equiv 0$,

4. $v\left(-f_{v}^{S}\right)=v\left(f_{0}^{2}+f_{3}^{2}+f_{2}^{2}\right) \equiv 0$.

Case (1): in this case we can find $n, m \in \mathbb{Z}$ such that $f_{0} \equiv n \pi$ and $f_{1} \equiv m \mathcal{\partial} \pi$. Equality 4.11 is equivalent to $\cos \left(f_{0}\right) \mu\left(-f_{v}^{S}\right)=\cos \left(-\partial f_{1}\right) \mu\left(f_{0}^{2}+f_{2}^{2}+f_{3}^{2}\right)$ which can also be written as

$$
\mu\left(f_{2}^{2}+f_{3}^{2}+n^{2} \pi^{2}\right)=(-1)^{n-m} \mu\left(-f_{2}^{2}-f_{3}^{2}-m^{2} \pi^{2}\right) .
$$

By setting $c=\left(n^{2}-m^{2}\right) \pi^{2}$ we infer from the proof of Proposition 3.3 (ii) that there exists $p \in \mathbb{Z}$ with $p \equiv n-m$ $(\bmod .2)$ such that $f_{2}^{2}+f_{3}^{2}=-\frac{\pi^{2}}{2}\left[\left(n^{2}+m^{2}\right) \pm p \sqrt{2 n^{2}-2 m^{2}-p^{2}}\right]$ if $2 n^{2}-2 m^{2}-p^{2} \geq 0$ while $f_{2}^{2}+f_{3}^{2}=$ $-\frac{\pi^{2}}{2}\left[\left(n^{2}+m^{2}\right) \pm J p \sqrt{2 m^{2}-2 n^{2}+p^{2}}\right]$ if $2 n^{2}-2 m^{2}-p^{2}<0$.

Case (2): the conditions on $f_{0}$ and $f_{0}^{2}+f_{2}^{2}+f_{3}^{2}$ entail we can find $n \in \mathbb{Z}$ and $m \in \mathbb{Z} \backslash\{0\}$ such that $f_{0} \equiv n \pi$ and $f_{0}^{2}+f_{2}^{2}+f_{3}^{2}=m^{2} \pi^{2}$, that is $f_{2}^{2}+f_{3}^{2}=\left(m^{2}-n^{2}\right) \pi^{2}$. Thus Equality 4.11 can also be written as $\mu\left(-f_{1}^{2}\right)=(-1)^{n-m} \mu\left(-f_{1}^{2}-\left(m^{2}-n^{2}\right) \pi^{2}\right)$. By setting $c=\left(n^{2}-m^{2}\right) \pi^{2}$, Proposition 3.3 (i) implies that there exists $p \in \mathbb{Z} \backslash\{0\}$ such that $p \equiv n-m(\bmod .2)$ and $-f_{1}^{2}=\left(\frac{p^{2} \pi-\left(n^{2}-m^{2}\right) \pi}{2 p}\right)^{2}$ which gives $f_{1}=\mathcal{J} \pi \frac{p^{2}+m^{2}-n^{2}}{2 p}$.

Case (3): the fact that $\sin \left(\mathcal{J} f_{1}\right) \equiv 0$ ensures that we can find $n \in \mathbb{Z}$ such that $f_{1} \equiv n \mathcal{J} \pi$, while $v\left(-f_{v}^{s}\right) \equiv 0$ gives that $-f_{1}^{2}-f_{2}^{2}-f_{3}^{2}=m^{2} \pi^{2}$ for a suitable $m \in \mathbb{Z} \backslash\{0\}$ and thus $-f_{2}^{2}-f_{3}^{2}=\left(n^{2}-m^{2}\right) \pi^{2}$. Again, by substituting the above relations we see that Equality 4.11 is equivalent to $\mu\left(f_{0}^{2}\right)=(-1)^{n-m} \mu\left(f_{0}^{2}+\left(m^{2}-n^{2}\right) \pi^{2}\right)$. By setting $c=\left(m^{2}-n^{2}\right) \pi^{2}$ we obtain that there exists $p \in \mathbb{Z} \backslash\{0\}$ such that $f_{0}=\frac{p^{2}+n^{2}-m^{2}}{2 p} \pi$.

Case (4): since $v\left(-f_{v}^{s}\right)=v\left(f_{0}^{2}+f_{3}^{2}+f_{2}^{2}\right) \equiv 0$, there exist $n, m \in \mathbb{Z} \backslash\{0\}$ such that

$$
f_{0}^{2}+f_{3}^{2}+f_{2}^{2}=n^{2} \pi^{2} \text { and }-f_{1}^{2}-f_{2}^{2}-f_{3}^{2}=m^{2} \pi^{2} .
$$

These relations imply $f_{1}^{2}=f_{0}^{2}-\left(n^{2}+m^{2}\right) \pi^{2}$, and thus Equality 4.11 becomes

$$
\mu\left(f_{0}^{2}\right)=(1)^{n-m} \mu\left(-f_{0}^{2}+\left(n^{2}+m^{2}\right) \pi^{2}\right) .
$$

By taking $c=\left(n^{2}+m^{2}\right) \pi^{2}$, by reasoning as in the proof of to Proposition 3.3 (ii) we obtain that there exists $p \in \mathbb{Z}$ with $p \equiv n-m$ (mod. 2) and such that $f_{0}=\frac{p \pm \sqrt{2\left(n^{2}+m^{2}\right)-p^{2}}}{2} \pi$ if $2\left(n^{2}+m^{2}\right)-p^{2} \geq 0$, while $f_{0}=$ $\frac{p \pm \mathcal{J} \sqrt{p^{2}-2\left(n^{2}+m^{2}\right)}}{2} \pi$ if $2\left(n^{2}+m^{2}\right)-p^{2}<0$. Straightforward computations then give that $f_{1}= \pm \mathcal{J} \frac{p \mp \sqrt{2\left(n^{2}+m^{2}\right)-p^{2}}}{2} \pi$ and $f_{2}^{2}+f_{3}^{2}=\frac{1}{2}\left[\left(n^{2}-m^{2}\right) \mp p \sqrt{2\left(n^{2}+m^{2}\right)-p^{2}}\right] \pi^{2}$ if $2\left(n^{2}+m^{2}\right)-p^{2} \geq 0$, while $f_{1}= \pm \mathcal{J} \frac{p \mp \mathcal{J} \sqrt{p^{2}-2\left(n^{2}+m^{2}\right)}}{2} \pi$ and $f_{2}^{2}+f_{3}^{2}=\frac{1}{2}\left[\left(n^{2}-m^{2}\right) \mp J p \sqrt{p^{2}-2\left(n^{2}+m^{2}\right)}\right] \pi^{2}$ if $2\left(n^{2}+m^{2}\right)-p^{2}<0$. 
Example 4.6. Let $\vartheta \in \mathcal{S} \mathcal{R}_{\mathbb{R}}(\Omega)$; the function $f=3 \pi+\partial \pi I+\pi \mathcal{J} \sqrt{5+2 \sqrt{3}} \cos \vartheta J+\pi \mathcal{J} \sqrt{5+2 \sqrt{3}} \sin \vartheta K$ is an example of case (iii) with $n=3, m=1$ and $p=2$, while $f=3 \pi+\mathcal{J} I+\pi J \sqrt{5} \cos \vartheta J+\pi J \sqrt{5} \sin \vartheta K$ is obtained with $n=3, m=1$ and $p=4$.

Example 4.7. Let $\vartheta \in \mathcal{S} \mathcal{R}_{\mathbb{R}}(\Omega)$; the function $f=\pi+3 \mathcal{J} \pi I+2 \sqrt{2} \pi \cos \vartheta J+2 \sqrt{2} \pi \sin \vartheta K$ is an example of case (iv) with $n=1, m=3$ and $p=2$, while $f=3 \pi-\partial \pi I+2 \sqrt{2} \pi \mathcal{\partial} \cos \vartheta J+2 \sqrt{2} \pi \partial \sin \vartheta K$ is obtained with $n=3$, $m=1$ and $p=2$.

Example 4.8. Let $\vartheta \in S \mathcal{R}_{\mathbb{R}}(\Omega)$; the function $f=-5 \pi+\partial \pi I+2 \sqrt{6} \pi \cos \vartheta J+2 \sqrt{6} \pi \sin 9 K$ is an example of case (v) with $n=1, m=5$ and $p=2$, while $f=7 \pi+5 \mathcal{\partial} \pi I+2 \sqrt{6} \pi \mathcal{\partial} \cos \vartheta J+2 \sqrt{6} \pi \mathcal{J} \sin 9 K$ is obtained with $n=5, m=1$ and $p=2$.

Conflict of interest: The author states no conflict of interest.

\section{References}

[1] A. Altavilla, Some properties for quaternionic slice-regular functions on domains without real points. Complex Var. Elliptic Equ. 60, n. 1 (2015), 59-77.

[2] A. Altavilla, C. de Fabritiis, *-exponential of slice-regular functions, Proc. Amer. Math. Soc. 147, 2019, $1173-1188$.

[3] A. Altavilla, C. de Fabritiis, s-Regular functions which preserve a complex slice, Ann. Mat. Pura Appl. (4) 197:4, 2018, 12691294.

[4] A. Altavilla, C. de Fabritiis, Sylvester operators on slice semi-regular functions, Linear Algebra Appl, 607C, 2020, 151-189, doi: 10.1016/j.laa.2020.08.009

[5] A. Altavilla, C. de Fabritiis, Applications of the Sylvester operator in the space of slice semi-regular functions, Concrete Operators, 7(1) 2020 1-12, doi: https://doi.org/10.1515/conop-2020-0001

[6] A. Altavilla, C. de Fabritiis, *-Logarithm for Slice Regular Functions, preprint arXiv: 2106.04227.

[7] A. Altavilla and G. Sarfatti. Slice-Polynomial Functions and Twistor Geometry of Ruled Surfaces in $\mathbb{C P}^{3}$. Math. Z. 291(3-4) (2019), 1059-1092.

[8] F. Colombo, J. Oscar Gonzalez-Cervantes, I. Sabadini, The C-property for slice regular functions and applications to the Bergman space, Compl. Var. Ell. Eq., 58, n. 10 (2013), 1355-1372.

[9] F. Colombo, I. Sabadini, D. C. Struppa, Entire Slice Regular Functions, SpringerBriefs in Mathematics, Springer, 2016.

[10] G. Gentili, J. Prezelij, F. Vlacci, On a defintion of logarithm of quaternionic function. arXiv:2108.08595.

[11] G. Gentili, D.C. Struppa, A new approach to Cullen-regular functions of a quaternionic variable, C. R. Acad. Sci. Paris, Ser. I 342 (2006).

[12] G.Gentili, D. C. Struppa, A new theory of regular functions of a quaternionic variable, Adv. Math. 216 (2007), no. 1, $279-301$.

[13] G. Gentili, C. Stoppato, D. C. Struppa, Regular Functions of a Quaternionic Variable, Springer Monographs in Matehmatics, Springer, 2013.

[14] R. Ghiloni, V. Moretti, A. Perotti, Continuous Slice Functional Calculus in Quaternionic Hilbert Spaces, Rev. Math. Phys. 25 (2013), 1350006-1-1350006-83.

[15] R. Ghiloni, A. Perotti, Slice regular functions on real alternative algebras, Adv. in Math., v. 226, n. 2 (2011), 1662-1691.

[16] R. Ghiloni, A. Perotti, C. Stoppato, The algebra of slice functions, Trans. of Amer. Math. Soc., Volume 369, N.7, 2017, pp.47254762.

[17] R. Ghiloni, A. Perotti, and C. Stoppato. Singularities of slice regular functions over real alternative *-algebras. Adv. Math., 305:1085-1130, 2017.

[18] R. Ghiloni, A. Perotti, C. Stoppato, Division algebras of slice functions, Proceedings A of the Royal Society of Edinburgh, 150(4):2055-2082 (2020), DOI:10.1017/prm.2019.13.

[19] K. Knopp, Theory and Application of Infinite Series, Hafner, New York 1951. 